\title{
VORSCHAU $02>$
}

\section{ENTWICKLUNG}

Restgassteuerung am Verbrennungsmotor mit variablen Steuerzeiten durch Zusatzventilhub

Reibungsreduzierung im Kolbensystem bei Pkw-Dieselmotoren

Weniger Reibung durch neue Kolbenringbeschichtung

\section{FORSCHUNG}

\section{Berechnungsverfahren zur Lebensdauerabschätzung von Abgassystemen}

Expandierende Faserschalen zur Dämmung heißgasführender Komponenten

\section{Emissionen von Biokraftstoffgemischen aus Motoren mit SCR-Katalysator}

Visualisierung der Temperatur- und Dampfverteilung in einem Benzinspray

Regelung der Schadstoffemissionen von Dieselmotoren

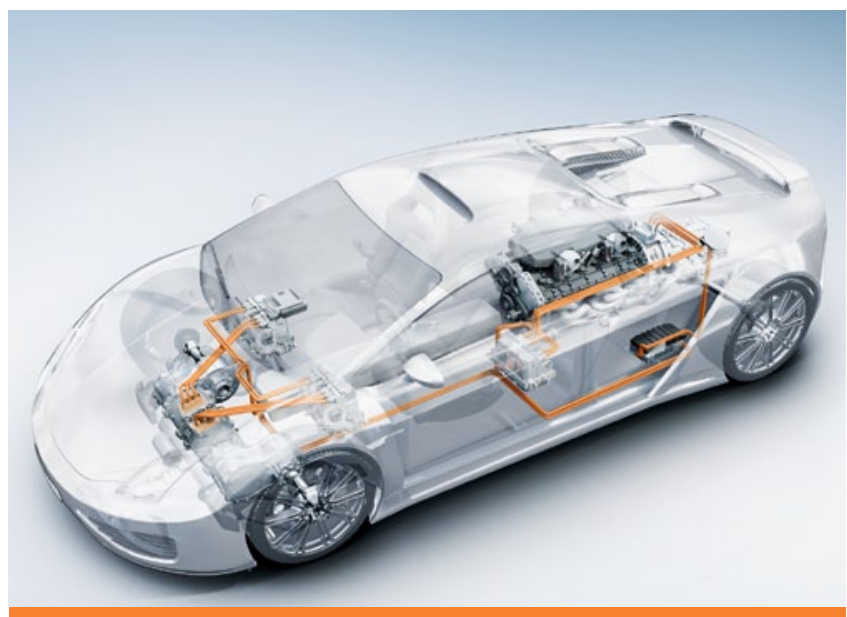

\section{TITELTHEMA}

\section{ELEKTRIFIZIERUNG DES ANTRIEBS}

Aktuell ist eine Vielzahl von elektrifizierten Antriebssträngen in der Entwicklung, sowohl als Hybridlösung als auch rein elektrisch. Dabei haben die Automobilhersteller, neben den grundsätzlichen technischen Herausforderungen, auch mit hohen Entwicklungs- und Bauteilkosten zu kämpfen. Um Entwicklungsaufwand zu sparen, müssen die OEMs und deren Zulieferer daher frühzeitig das am besten geeignete Elektrifizierungskonzept aus der Fülle der Möglichkeiten auswählen und in einen Produktentstehungsprozess hin zur Serienfertigung überführen. Um darüber hinaus qualitativ hochwertige Systeme zu vertretbaren Kosten darstellen zu können, gilt es Skaleneffekte zu schaffen.

Für beide Herausforderungen zeigen wir in der MTZ Lösungsansätze: Bosch hat eine domänenübergreifende Simulationsumgebung für die Entwicklung von Hybridantriebssträngen erarbeitet. Der Ansatz lässt sich durchgängig von der Konzeptphase und Systemauslegung bis hin zur Fahrzeugapplikation einsetzen. Die erste Anwendung erfolgte bei der Auslegung eines Hybridsystems für einen Sportwagen. Volkswagen wiederum hat einen modularen Baukasten entwickelt, dessen Komponenten den Aufbau unterschiedlich elektrifizierter Antriebssysteme für Hybrid- und Elektrofahrzeuge des Konzerns ermöglichen. In den neuen, rein elektrisch betriebenen Fahrzeugen e-up! und e-Golf von Volkswagen findet der Baukasten das erste Mal Verwendung. Wie vergleichsweise kleine Hersteller mit der Herausforderung der Elektrifizierung des Antriebs umgehen, erläutert exemplarisch Pete Richings, Hybrids and Electrification Director Jaguar Land Rover, im Interview mit der MTZ. 